\title{
Effect of Variable Emittance Coatings on the Operation of a Miniature Loop Heat Pipe
}

\author{
Donya M. Douglas ${ }^{1}$, Jentung $\mathrm{Ku}^{1}$, Laura Ottenstein ${ }^{1}$, \\ Theodore Swanson ${ }^{2}$, Steve Hess ${ }^{3}$, Ann Darrin ${ }^{4}$ \\ ${ }^{1}$ Thermal Engineering Branch, MS 545, NASA Goddard Space Flight Center, Greenbelt MD 20771 \\ ${ }^{2}$ Mechanical Systems Division, MS 540, NASA Goddard Space Flight Center, Greenbelt MD 20771 \\ ${ }^{3}$ Sensortex, Inc. 515 Schoolhouse Road, Kennett Square, PA 19348 \\ ${ }^{4}$ The Johns Hopkins University Applied Physics Lab, Laurel MD 20723 \\ (301)286-6952,Donva.M.Douglas@gsfc.nasa.gov
}

\begin{abstract}
As the size of spacecraft shrink to accommodate small and more efficient instruments, smaller launch vehicles, and constellation missions, all subsystems must also be made smaller. Under NASA NRA 03-OSS-02, Space Technology-8 (ST 8), NASA Goddard Space Flight Center and Jet Propulsion Laboratory jointly conducted a Concept Definition study to develop a miniature loop heat pipe (MLHP) thermal management system design suitable for future small spacecraft. The proposed MLHP thermal management system consists of a miniature loop heat pipe (LHP) and deployable radiators that are coated with variable emittance coatings (VECs). As part of the Phase A study and proof of the design concept, variable emittance coatings were integrated with a breadboard miniature loop heat pipe. The miniature loop heat pipe was supplied by the Jet Propulsion Laboratory (JPL), while the variable emittance technology were supplied by Johns Hopkins University Applied Physics Laboratory and Sensortex, Inc. The entire system was tested under vacuum at various temperature extremes and power loads. This paper summarizes the results of this testing and shows the effect of the VEC on the operation of a miniature loop heat pipe.
\end{abstract}

\section{INTRODUCTION}

In response to an Office of Space Science NRA, Goddard Space Flight Center in conjunction with the Jet Propulsion Laboratory conducted a study to develop a thermal control system suitable for micro and nano-satellites. The proposed miniature loop heat pipe (MLHP) Thermal Management System consists of a loop heat pipe (LHP) with multiple evaporators and multiple condensers, and deployable radiators coated with variable emittance coatings (VECs). Other key elements include thermal electrical coolers (TECs) on the LHP compensation chamber (CC), a capillary flow regulator at the downstream of the condensers, and an aluminum coupling block between the vapor line and liquid line. For the ST8 flight validation, an MLHP consisting of two evaporators, two condensers, one body mounted radiator and one deployable radiator will be used, as shown schematically in Figure 1.

The loop heat pipe, which is a two-phase thermal device that utilizes surface tension force developed in a capillary wick to drive the fluid around the loop, was supplied by the Jet Propulsion Laboratory (JPL), while the variable emittance coating, a smart coating that autonomously varies it's effective emittance, was supplied by Sensortex, Inc. The loop operates like a conventional LHP in that as the fluid circulates in the loop, heat is still transported from the evaporator to the condenser, and the compensation chamber (CC) establishes the temperature of the system. However, the application of the VEC to the condenser offers added benefits, which is the topic of this paper. 


\section{OPERATING PRINCIPLES}

\section{MLHP System with VEC}

Even with the dual evaporator and condensers, the functionality of the LHP remains the same; surface tension force developed in the capillary wick drives the fluid around the loop and the LHP must be successfully started before the thermal subsystem can begin to service. Once the loop has started, the instruments can be turned on and dissipate heat to the evaporators. The evaporators can take even or uneven heat loads. Likewise, the condenser sinks can be at even or uneven temperatures. The loop will provide a single operating temperature for all instruments. When an instrument is turned off, the attached evaporator will work as a condenser and receive vapor from other evaporators attached to the "on" instruments, maintaining all instruments at near the saturation temperature. The evaporators can automatically switch between evaporating and condensing modes based on the surrounding thermal conditions. Thus, each instrument can operate independently in any fashion without regard to other instruments. The LHP can also adapt to rapid changes in the instrument heat outputs.

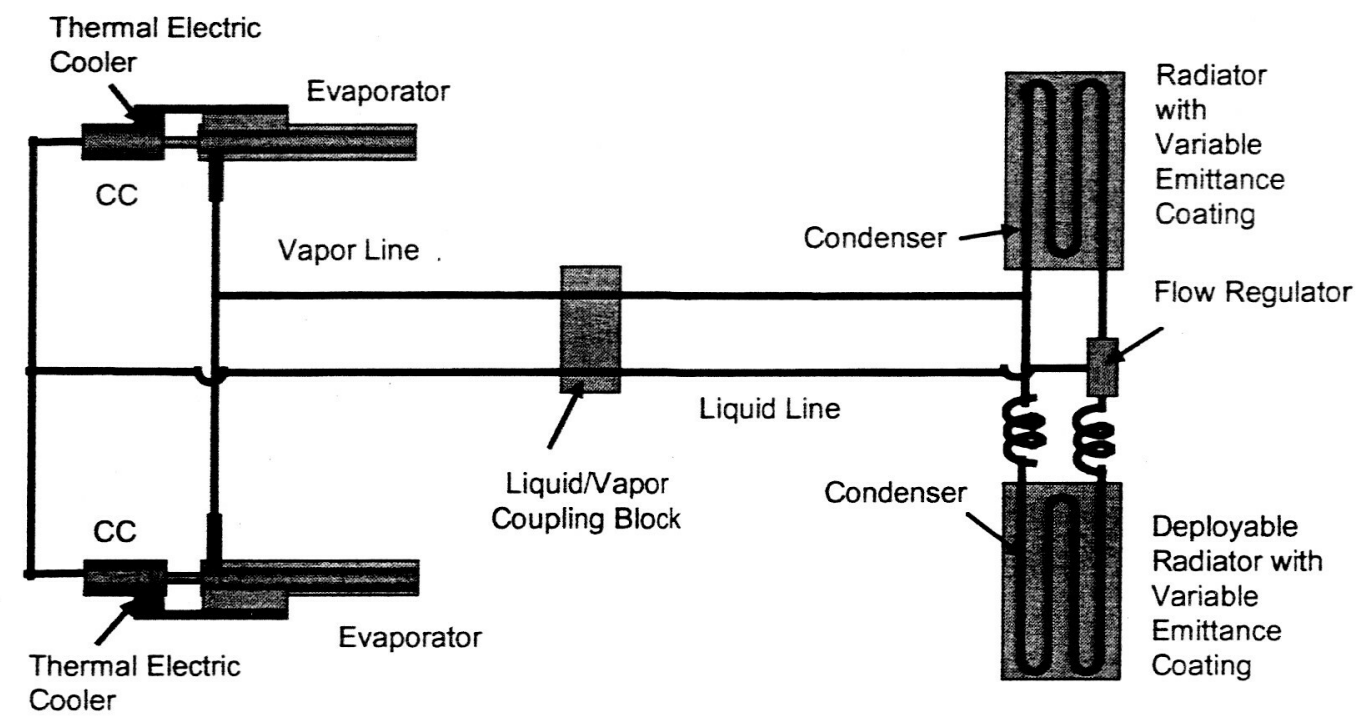

FIGURE 1. Schematic of the Proposed MLHP Thermal Subsystem

Each of the multiple, parallel condensers will receive an appropriate mass flow rate based on its thermal environment and the total system heat load so that the conservation laws of mass, momentum and energy are satisfied within the condenser section. Any changes in the system heat load and/or condenser sink environments will result in a redistribution of flow rates among the condensers so that the conservation laws are satisfied. When a condenser is fully utilized, vapor exiting the condenser will be stopped by the flow regulator and the excess vapor will be diverted to other condensers. As long as the radiators as a whole can dissipate the total system heat load, some of the radiators can even be exposed to environments that are hotter than the loop saturation temperature. Liquid freezing in the condensers during the survival mode can be prevented by setting the VECs to the minimum emittance, and use supplemental heaters if needed.

When the total heat load exceeds the LHP heat transport capability, vapor will penetrate the wick and flow to the CC. The loop operating temperature will rise. Tests results indicate that, in most cases, the LHP will reach a new steady state at a higher saturation temperature. Thus, the LHP will undergo a graceful degradation in performance rather than a catastrophic failure. When the heat load is reduced, the loop will recover and operate at the original set point temperature.

The flow circulation in the LHP will stop when the evaporator temperature drops below the CC saturation temperature. Thus, when all instruments are turned off, the loop will automatically shutdown if the CC saturation 
temperature is maintained. Thus, the LHP works as a thermal switch and no heat will be transmitted from the instruments to the radiators.

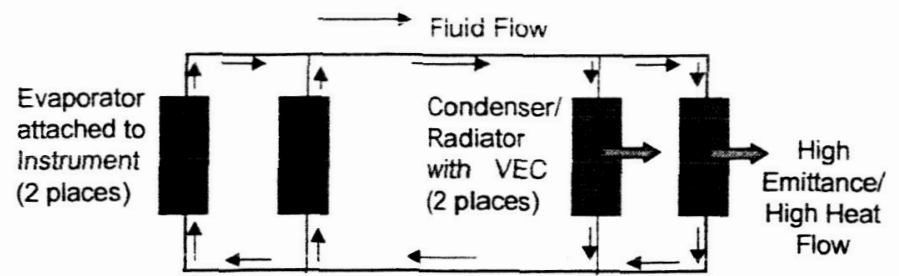

(a) Normal Operation (both instruments on)

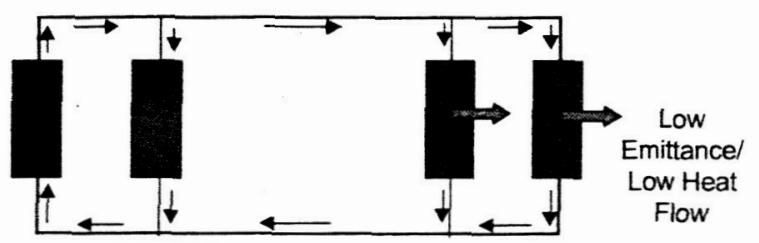

(b) Heat Load Sharing (one instrument on, one off)

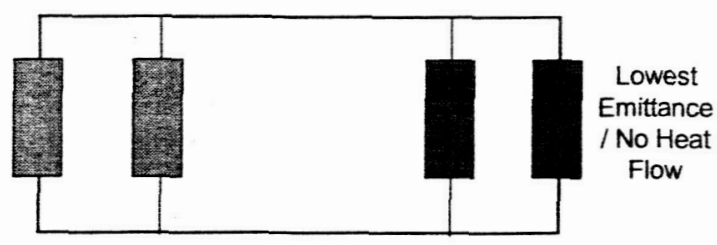

(c) Survival Mode (both instruments off)

FIGURE 2. Operating Modes of MLHP

Operation of the MLHP Thermal Management System can be illustrated in Figure 2, using an LHP with two evaporators and two condensers as an example. There are three basic operating modes after the loop has been started:

- Both instruments are turned on and a high heat rate is flowing to the radiators. The VECs are commanded to a high or medium emittance state depending on the radiator sink temperatures.

- One instrument is turned on and the other is turned off. Part of the vapor generated in the evaporator attached to the 'on" instrument will flow to the evaporator of the 'off' instrument, i.e. the "off' instrument becomes a heat sink. The remaining heat load will flow to the condensers, and the VECs are commanded to a medium or low emittance state depending on the radiator sink temperatures.

- Both instruments are turned off. The spacecraft or the instruments are in a survival mode. The MLHP is shut down and becomes a thermal switch automatically. No heat is transmitted from the instruments to the radiators through the MLHP. The VECs are commanded to the lowest emittance so as to help prevent liquid freezing.

\section{Variable Emittance Coating}

The VEC technology proposed for the MLHP Thermal Management is being developed by Sensortex, Inc. located in Kennett Square, Pennsylvania. This technology addresses the active control of thermal radiation with a new and novel approach that eliminates the cost and complexity of existing methods. The ESR uses the fact that a very high emissivity surface, $(\varepsilon \sim 1)$, in good thermal contact with a low emissivity surface, will reach the same temperature. 
When this occurs, the apparent emissivity of the system (skin plus cover) approaches 1 . However, if the thermal contact is poor, the front surface with the high emissivity film can only radiate the energy it absorbs. In equilibrium, the cover will be at a lower temperature since the absorbed energy, controlling its temperature, comes from a low emissivity surface on the base surface. The amount of energy radiated by the system (cover + skin) is now lower. Thus by switching between these states of good and poor contact between the high emissivity film and the spacecraft skin, the radiation from the skin (which can be treated as an effective emissivity) can be controlled over very wide limits.

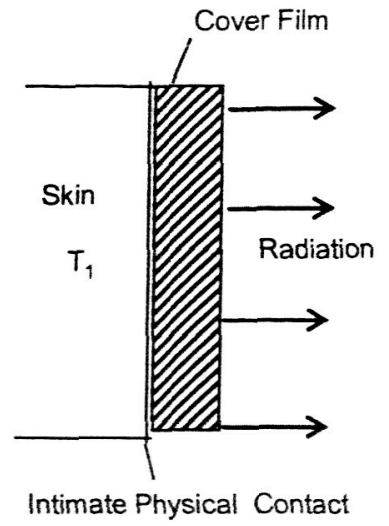

High Radiation (On State)

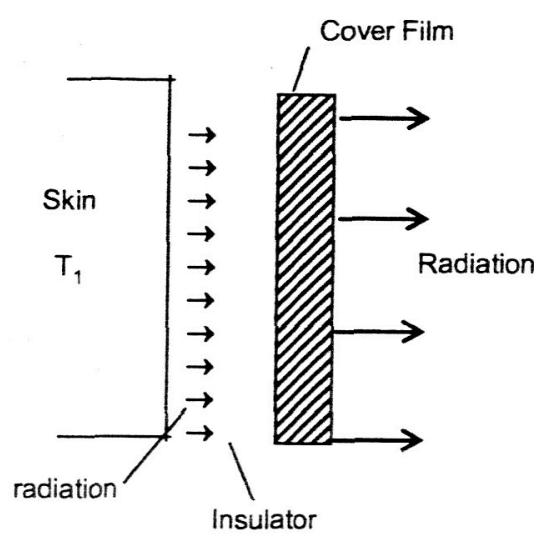

Low Radiation (Off State)

FIGURE 3. ElectroStatic VEC

To operate properly, the ESR must be capable of achieving intimate thermal contact between the skin and the outer surface. This contact is established by an electrostatic (capacitance-like) hold-down so that the system, consisting of the low emissivity surface with the high emissivity cover, will have a high emissivity when a voltage is applied. Alternately, with the voltage removed, this cover can relax or spring away, thus destroying this thermal contact and resulting in a lowered effective emissivity. When attracted, it makes good conductive thermal contact so the emitting surface of the cover is at the skin temperature. The outer surface of the cover has a high emissivity, so the skin has a high effective emissivity $(\sim 1)$. In the released state, the heat transfer mechanism is now only by radiation, so the only energy that the outer cover can radiate (at equilibrium) is that radiated from the skin, which is fabricated with a low emissivity. The emissivity of the outer skin doesn't change, however its temperature drops and the result is a drop in the radiated energy.

Control of the ESR has the characteristics of low power consumption and simple control circuitry. ESR films have been demonstrated to operate effectively with moderate levels of applied DC voltages (i.e. $200-500 \mathrm{~V}$ ). Application of the film control voltage results in the high emissivity state. Removal of (i.e. shorting) the film control voltage gives the low emittance state. It should be noted that the ESR device basically operates as a high quality capacitor (C $\sim 400 \mathrm{pfd} / \mathrm{cm}^{2}$ ). If the control voltage is simply removed (as opposed to shorted) from the ESR film, the high emissivity state will remain for a limited time. Experimental measurements have been conducted to measure the power radiated to a cold background in a vacuum environment. GSFC have measured changes in effective emissivity as high as 0.3 while Sensortex have measured changes as high as 0.7 with and without applied voltage. However the planned device for the ST-5 will likely settle at the median of approximately 0.5 .

\section{MLHP BREADBOARD SYSTEM OVERVIEW}

The MLHP Breadboard, shown in Figures 3, is charged with 15.5 grams of anhydrous ammonia. It consists of two evaporators, two condensers, a common vapor transport line, a common liquid return line, and four VEC substrates. Each evaporator, which is made of $15 \mathrm{~mm}$ outside diameter (O.D.) aluminum tubing by $76.2 \mathrm{~mm}$ long, has its own integral compensation chamber (CC). Each CC is made of stainless steel tube of $14.8 \mathrm{~mm}$ O.D. x $81.8 \mathrm{~mm}$ long. 
One evaporator has a titanium wick with a pore radius of about $3 \mu \mathrm{m}$, while the other has a nickel wick with a pore radius of about $0.5 \mu \mathrm{m}$. A thermal mass of 500 grams was attached to each evaporator to simulate the instrument mass. Two cartridge heaters were attached to each thermal mass to provide heat loads between $1 \mathrm{~W}$ and $200 \mathrm{~W}$ per evaporator. In order to demonstrate heat load sharing, each thermal mass had two holes to accommodate two passes for the coolant flow. In addition, each thermal mass was designed to provide a flat surface with an area of $76 \mathrm{~mm}$ by $300 \mathrm{~mm}$ so that it could be cooled by radiation during heat sharing mode in the TV test.

The vapor line and liquid line, each $1168 \mathrm{~mm}$ long, are made of stainless steel tube with an O.D. of $3.3 \mathrm{~mm}$ and 2.2 $\mathrm{mm}$, respectively. The vapor and liquid lines branch out to feed into the two evaporators and two condensers. Each condenser is made of stainless steel tube of $2.2 \mathrm{~mm}$ O.D. x $762 \mathrm{~mm}$ long. A flow regulator consisting of capillary wicks is installed at the downstream of the condensers. A Thermo-Electric Cooler (TEC) was installed on each CC through a copper saddle. The hot side of the TEC was connected to the evaporator through a copper strap. Each TEC was controlled by a bi-polar power supply. Changing the polarity on the power supply changed the TEC operation between heating and cooling modes.

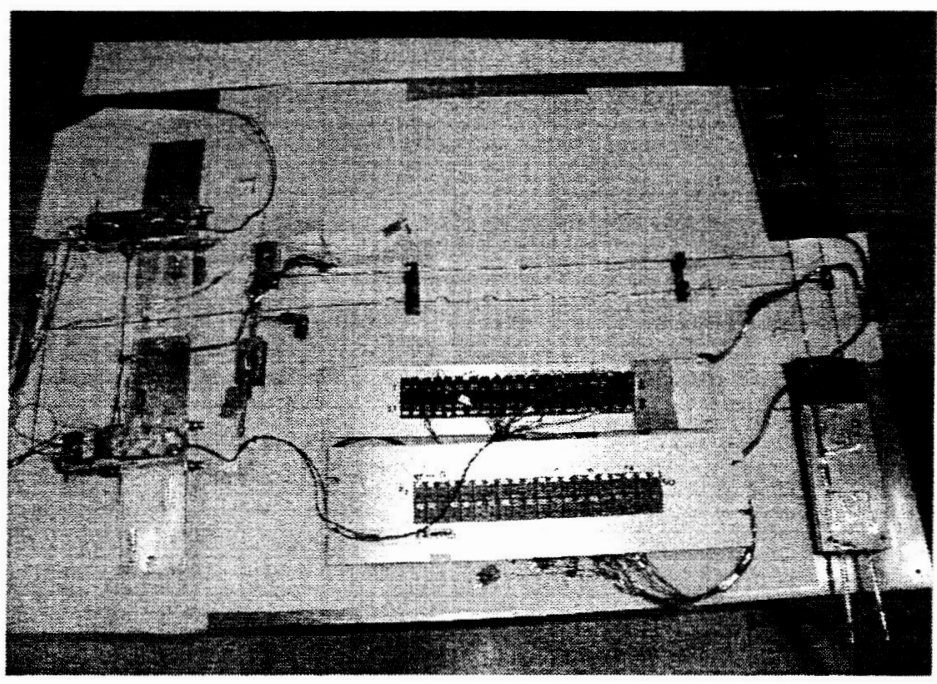

FIGURE 4. Photograph of MLHP Breadboard

Four VEC substrates, each had a dimension of $82.6 \mathrm{~mm} \times 177.8 \mathrm{~mm}$, were attached to the Condenser 1 cold plate, two at the top and two at the bottom. One of the VEC substrates, provide by APL, was of a louver shutter design. The other three were electrostatic VECs and were provided by Sensortex, Inc. These VEC substrates were relatively small and could dissipate only $20 \mathrm{~W}$ at the maximum emittance. The budget and schedule constraints in the Study Phase prevented the production and testing of more VEC substrates. A heater was attached to the underside of one VEC substrate. During the survival mode test, the radiator was exposed to different cryopanel temperatures and the VECs were set to their maximum and minimum emittances. The heater power required to maintain the condenser above the freezing point of the working fluid was measured for each case.

An aluminum plate of $533 \mathrm{~mm} \times 438 \mathrm{~mm}$ by $3.18 \mathrm{~mm}$ thick was attached to the Condenser 2 cold plate to serve as the radiator. This radiator was painted black on both sides and was the main heat dissipating element during the TV test. The flat surface of each thermal mass attached to the evaporator was covered with Kapton tape for heat load sharing operation.

Six copper cryopanels were used as radiator sinks, two for each condenser/radiator and one for each thermal mass. Each cryopanel was painted black on one side and contained electrical heaters and copper tubes for liquid nitrogen on the other side. The electrical heaters and liquid nitrogen flow allowed the panel temperature to be changed quickly. The cryopanels facing the thermal mass were maintained near the $\mathrm{CC}$ saturation temperature during normal 
operation so that no heat gain or loss would occur. One of the cryopanels was maintained at a temperature below the saturation during heat load sharing tests.

Ninety Type T thermocouples, shown in Figure 5, were used to monitor the temperatures. A data acquisition system (DAS) consisting of a personal computer, a CRT monitor, and two dataloggers was used to display and store test data every two seconds.

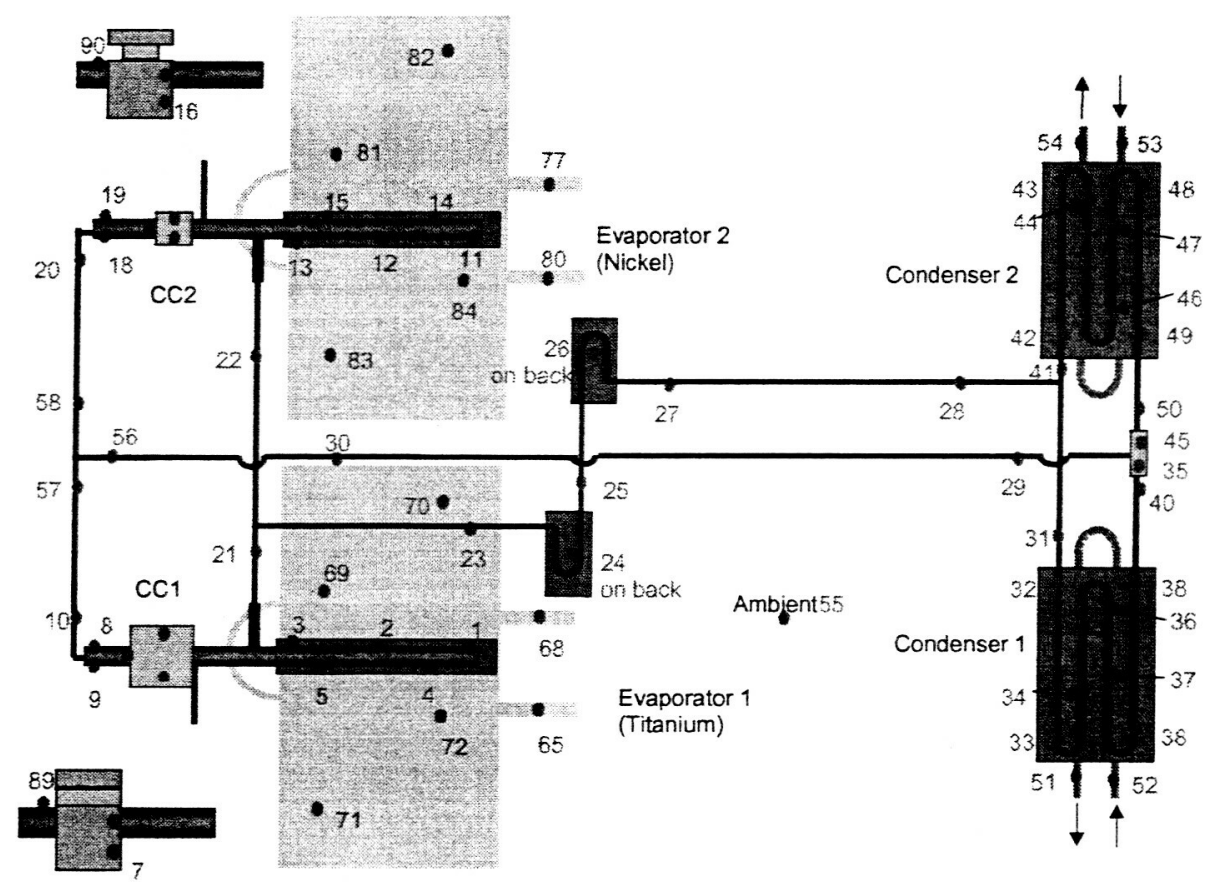

FIGURE 5. Schematic of MLHP Breadboard with Thermocouple Locations

\section{THERMAL VACUUM TEST RESULTS}

The main objective of the TV Test was to demonstrate that the VECs could regulate the temperature of the liquid exiting the condenser and regulate the radiator heat dissipation during the survival mode.

Figure 6 shows the results of the VEC Effects Test that was run on April 2, 2004. The objective of the test was to determine the temperature of liquid leaving Condenser 1 as a function of the VEC emittance at two different heat loads. During this test, all cryopanels for Condensers 1 and Condenser 2 were kept at $120 \mathrm{~K}$. Initially, the system power was set to 30 watts and the VEC state was switched between low and high emittance. Then the power was set to $20 \mathrm{~W}$ and again the VECs were switched between high and low emittance states. At 1200 hours the Compensation Chamber, Condenser inlet, and Condenser outlet temperatures were all around $305 \mathrm{~K}$ with 30 watts on the evaporators and the VECs in the low emittance state. At approximately 1230 hours, the VECs were set in the high emittance state. This resulted in higher heat rejection and a decrease in the Condenser outlet temperature. At 1330 hours the slope of the temperature drop increased further as the Evaporator power was decreased to $20 \mathrm{~W}$ and the VECs remained in the high emittance state. When the VECs were switched to the low emittance state, the Condenser outlet temperature started increasing, showing a clear positive effect of the VECs. The liquid temperature at the Condenser exit is directly related to the subccoling to be overcome by the CC supplemental heaters. The results showed that the amount of control heater power required by the TEC is reduced when the VECs 
are in the low emittance state. Unfortunately, because of the limited sizes of the VEC radiators, the heater power savings could not be precisely determined. Table 1 summarizes the results of this test.

ST8 LHP 2 April 2004

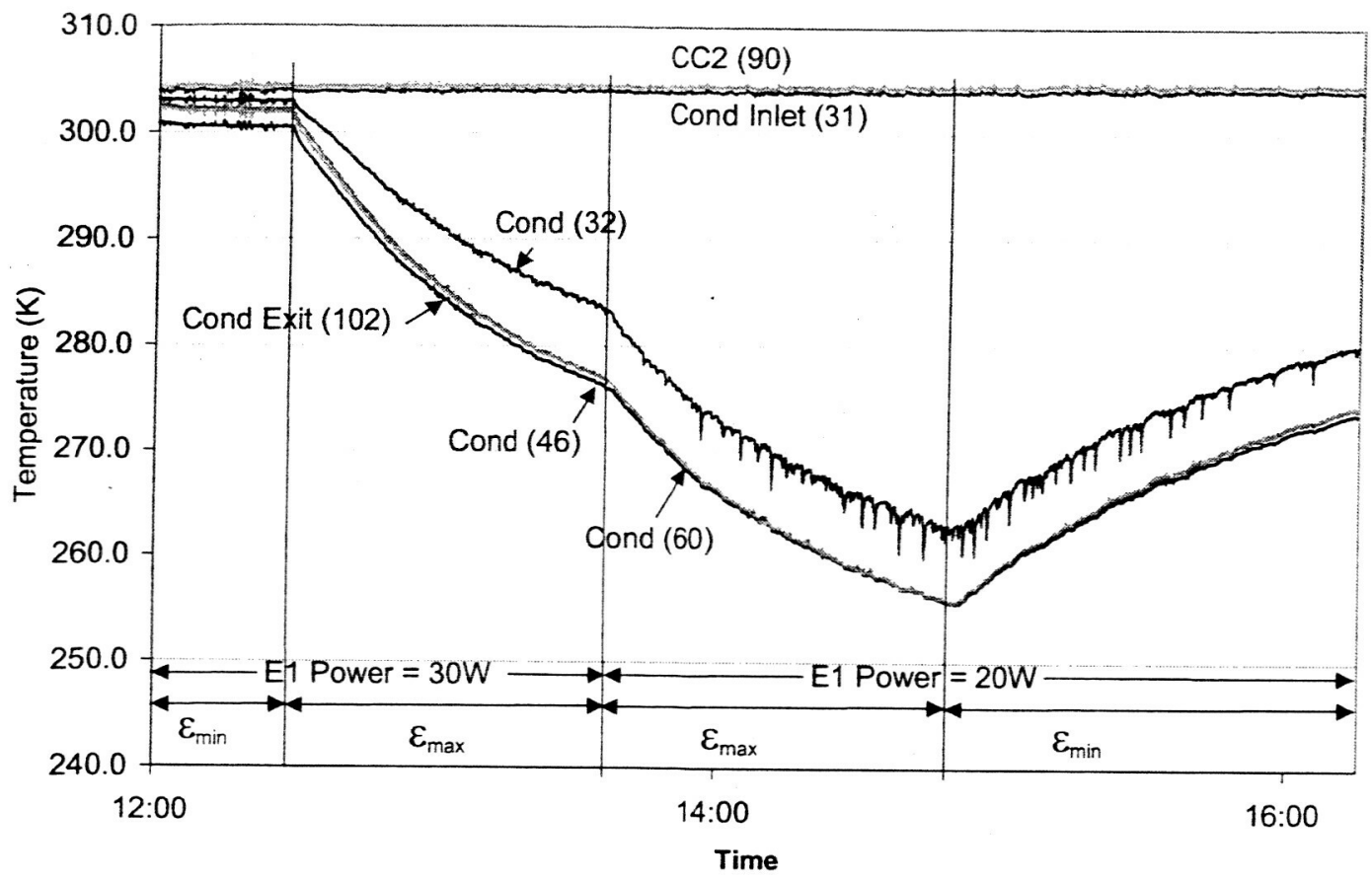

FIGURE 6. VEC Effect on Condenser Exit Temperature

TABLE 1. VEC Effect on Condenser Exit Temperature

\begin{tabular}{lcc}
\hline System Heat Load & VEC Emittance & Temperature of Liquid Leaving Condenser 1 \\
\hline $30 \mathrm{~W}$ & Max & $275 \mathrm{~K}$ \\
$30 \mathrm{~W}$ & Min & $300 \mathrm{~K}$ \\
$20 \mathrm{~W}$ & Max & $254 \mathrm{~K}$ \\
$20 \mathrm{~W}$ & Min & $273 \mathrm{~K}$ \\
\hline
\end{tabular}

Tests were also performed to demonstrate the effectiveness of the VECs in reducing the supplemental heater power required in a simulated survival mode. No heat loads were applied to the Evaporators and the loop was shut down by keeping the Compensation Chamber temperature of Evaporator 1 at $303 \mathrm{~K}$. The first test was conducted by holding the Condenser 1 cryopanel at $180 \mathrm{~K}$. The bonding material for the VEC substrates limited testing to a minimum temperature of $223 \mathrm{~K}$. As a result, when the Condenser 1 temperature reached $230 \mathrm{~K}$, the heater on the VEC substrate was turned on. Measurements were made to determine the required heater power to keep the Condenser 1 temperature at $230 \mathrm{~K}$ with the VECs at their maximum and minimum emittances. The same tests were repeated for cryopanel temperature of $120 \mathrm{~K}$. Test results, which are summarized in Table 2, showed that the required heater power was reduced by more than one half as the emittance was changed from the maximum to the minimum. Optimizing the VEC design or substrate would result in larger heater power savings.

Table 2. Power Requirement to Maintain Condenser 1 Radiator at $230 \mathrm{~K}$

\begin{tabular}{lcc}
\hline Cryopanel Temperature & Emittance & Heater Power to Radiator \\
\hline $180 \mathrm{~K}$ & Max & $7.6 \mathrm{~W}$ \\
$180 \mathrm{~K}$ & Min & $3.2 \mathrm{~W}$
\end{tabular}




\section{CONCLUDING REMARKS}

The MLHP Breadboard was tested under thermal vacuum conditions. The VECs were shown to provide heater power savings during survival mode and a reduction in the power required to maintain the compensation chamber temperature at the control setpoint. The integrated system retained all the performance characteristics of state-ofthe-art LHPs and offers additional advantages to enhance the functionality, performance, versatility, and reliability of the system, including:

- flexible locations of instruments and radiators

- a single interface temperature for multiple instruments

- cooling the "on" instruments and warming the "off" instruments simultaneously

- improving start-up success, maintaining a constant LHP operating temperature over a wide range of instrument powers

- effecting automatic thermal switching and thermal diode actions

- reducing supplemental heater powers

Potential applications of the MLHP Thermal Management System for future missions include small satellites for remote sensing of the environment, astronomical probing for studying the solar system, and Mars rover.

\section{NOMENCLATURE}

APL $=$ Applied Physics Laboratory

$\mathrm{CC}=$ Compensation Chamber

DAS $=$ Data Acquisition System

$\varepsilon=$ emittance

$\mathrm{ESR}=$ ElectroStatic Radiator

GSFC $=$ Goddard Space Flight Center

JHU $=$ Johns Hopkins University

$\mathrm{JPL}=$ Jet Propulsion Laboratory

$\mathrm{K}=$ Kelvin

LHP = Loop Heat Pipe

MLHP = Minature Loop Heat Pipe

NMP $=$ New Millennium Program

O.D. $=$ Outside Diameter

ST $-5=$ Space Technology 5

ST $-8=$ Space Technology 8

$\mathrm{TC}=$ Thermocouples

TEC $=$ Thermo-Electric Cooler

VEC $=$ Variable Emittance Coatings

$\mathrm{W}=$ Watts

\section{REFERENCES}

Douglas, D., Michalek, T. and Swanson, T. D., "Design of the Thermal Control System for the Space Technology 5 Microsatellite," $31^{\text {st }}$ International Conference on Environmental Systems 2000-01-2214, 2001.

Ku, J. and G. Birur, "Testing of a Loop Heat Pipe with Two Evaporators and Two Condensers," SAE Paper \# 2001-01-2190, 2001. 